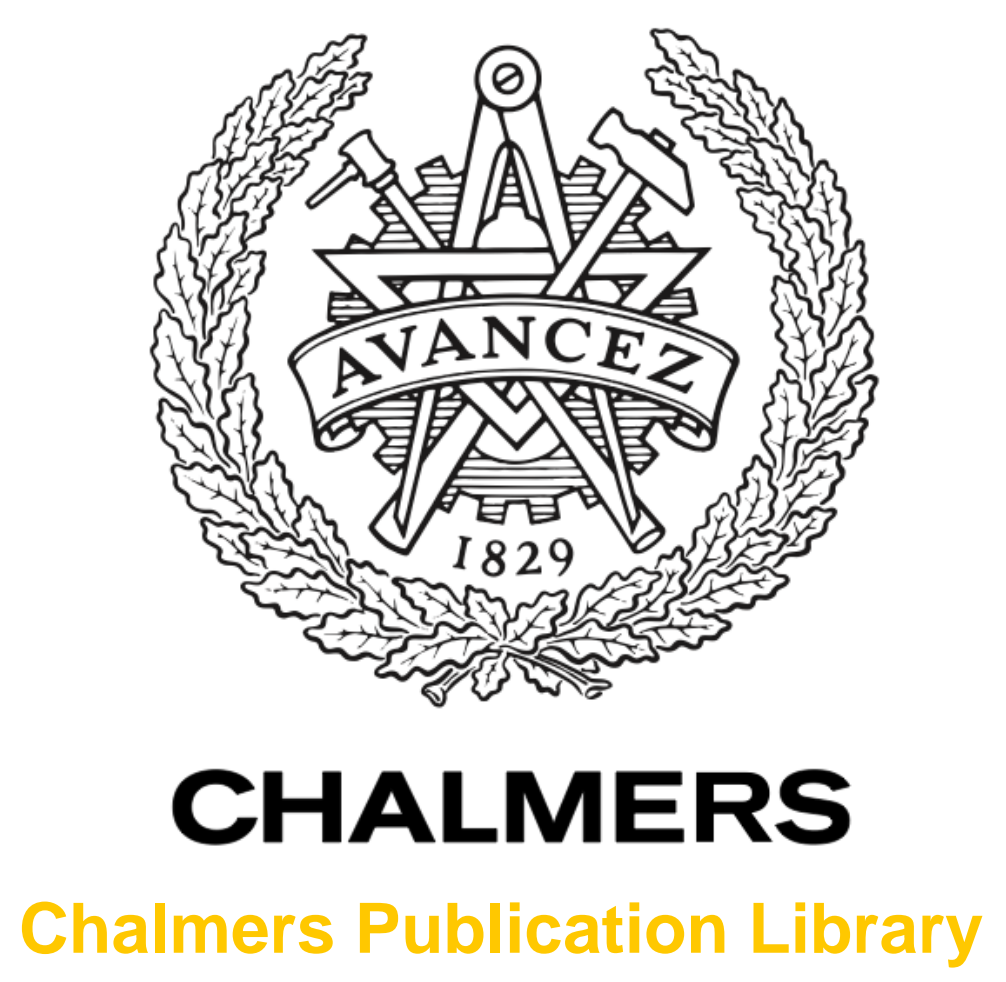

Interpolation based on stationary and adaptive AR(1) modeling

This document has been downloaded from Chalmers Publication Library (CPL). It is the author's version of a work that was accepted for publication in:

36th IEEE International Conference on Acoustics, Speech, and Signal Processing, ICASSP 2011; Prague; 22 May 2011 through 27 May 2011 (ISSN: 15206149)

Citation for the published paper:

Johansson, E. ; Ström, M. ; Svensson, L. (2011) "Interpolation based on stationary and adaptive AR(1) modeling". 36th IEEE International Conference on Acoustics, Speech, and Signal Processing, ICASSP 2011; Prague; 22 May 2011 through 27 May 2011 pp. $4052-$ 4055.

Downloaded from: http://publications.lib.chalmers.se/publication/146866

Notice: Changes introduced as a result of publishing processes such as copy-editing and formatting may not be reflected in this document. For a definitive version of this work, please refer to the published source. Please note that access to the published version might require a subscription.

Chalmers Publication Library (CPL) offers the possibility of retrieving research publications produced at Chalmers University of Technology. It covers all types of publications: articles, dissertations, licentiate theses, masters theses, conference papers, reports etc. Since 2006 it is the official tool for Chalmers official publication statistics. To ensure that Chalmers research results are disseminated as widely as possible, an Open Access Policy has been adopted.

The CPL service is administrated and maintained by Chalmers Library. 


\title{
INTERPOLATION BASED ON STATIONARY AND ADAPTIVE AR(1) MODELING
}

\author{
Eija Johansson, Marie Ström, Mats Viberg and Lennart Svensson \\ Department of Signals and Systems, Signal Processing Group \\ Chalmers University of Technology \\ Gothenburg, Sweden
}

\begin{abstract}
In this paper, we describe a minimal mean square error (MMSE) optimal interpolation filter for discrete random signals. We explicitly derive the interpolation filter for a first-order autoregressive process $(\mathrm{AR}(1))$, and show that the filter depends only on the two adjacent points. The result is extended by developing an algorithm called local AR approximation (LARA), where a random signal is locally estimated as an AR(1) process. Experimental evaluation illustrates that LARA interpolation yields a lower mean square error than other common interpolation techniques, including linear, spline and local polynomial approximation (LPA).
\end{abstract}

Index Terms - Interpolation, LMMSE estimation, autoregressive modeling, adaptive filtering

\section{INTRODUCTION}

When sampling signals, a traditional assumption is that the sampling frequency must be higher than twice the signal bandwidth (the Shannon-Nyquist rate), in order to avoid aliasing. However, recent studies have shown that this is an unnecessary limitation and provide ways to sample a signal below this rate and compensate for the aliasing that occurs [1], [2].

Matthews [3] has studied the problem of designing a filter to reconstruct a continuous signal that has been sampled below the Shannon-Nyquist rate. However, since most signals are stored and processed in digital format, a densely sampled signal is often a sufficient representation of a continuous signal. Furthermore, a digital to digital converter is easier to implement than an analogue to digital converter. In this paper, we investigate the reconstruction of a discrete random signal by interpolating a downsampled version of the signal. Reconstructions of this nature have diverse applications including biomedical imaging [4] and audio processing [5].

For a discrete random signal with known spectrum, we derive the optimal interpolation filter in a minimum mean square error (MMSE) sense. Based on statistical information about the spectrum of the signal, the filter is designed to compensate for the aliasing and to retain the statistical properties of the signal. We derive an explicit form of this filter for a stationary first-order autoregressive process $(\mathrm{AR}(1))$. The resulting filter is then extended to a general interpolation algorithm, that can be used on a larger set of signals. This is done by approximating the signal locally as an AR(1) process, where the $\mathrm{AR}(1)$ parameter is estimated from the data without prior information about the original signal. We name this algorithm local AR approximation (LARA).

To evaluate the performance of LARA interpolation, we compare it with other interpolation techniques: linear, spline and local polynomial approximation (LPA) [6] interpolation.

\section{PROBLEM STATEMENT}

Consider a wide-sense stationary, discrete signal $x[n]$, which is sampled into

$$
x_{\mathrm{d}}[n]=x[n L],
$$

where $L$, the downsampling factor, is a positive integer. Our objective is to reconstruct $x[n]$ from $x_{\mathrm{d}}[n]$, even though aliasing has occurred. We limit our solution to the set of linear reconstructions, and aim to minimise the MSE within this set. When interpolating a discrete signal, the optimal interpolation filter is a digital multirate filter, since for each sample the filter yields several interpolation points. By dividing the filter into $L$ parallel filters, where $L$ is the upsampling factor, the multirate filter can be represented by a bank of single-rate filters.

Let us therefore introduce the filters $H_{\mathrm{k}},(k=0, . ., L-1)$, where $H_{k}$ reconstructs samples of the form

$$
x_{\mathrm{dk}}[n] \equiv x[n L+k] .
$$

For each of these interpolation filters, the optimal filter, in the MMSE sense, is a Wiener filter [7]:

$$
H_{\mathrm{k}}\left(e^{j \Omega_{\mathrm{d}}}\right)=\frac{P_{x_{\mathrm{d}} x_{\mathrm{dk}}}\left(e^{j \Omega_{\mathrm{d}}}\right)}{P_{x_{\mathrm{d}} x_{\mathrm{d}}}\left(e^{j \Omega_{\mathrm{d}}}\right)},
$$

where $\Omega_{\mathrm{d}}$ is the angular frequency in radians/sample, $P_{x_{\mathrm{d}} x_{\mathrm{d}}}$ is the spectrum of $x_{\mathrm{d}}$ and $P_{x_{\mathrm{d}} x_{\mathrm{dk}}}$ is the cross spectrum of $x_{\mathrm{d}}$ and $x_{\mathrm{dk}}$. Expressing the cross spectrum in terms of the spectrum of $x[n]$ results in the following proposition,

Proposition 1. Let a random signal $x[n]$ with known spectrum $P_{x x}$ be sampled into $x_{\mathrm{d}}[L n]$ with spectrum $P_{x_{\mathrm{d}} x_{\mathrm{d}}}$. Then the MMSE optimal interpolation filter to reconstruct $x$ from $x_{\mathrm{d}}$ is given by

$$
H_{\mathrm{k}}\left(e^{j \Omega_{\mathrm{d}}}\right)=\frac{\frac{e^{j \frac{\Omega_{\mathrm{d}} k}{L}}}{L} \sum_{n=<L>} e^{-j \frac{n 2 \pi k}{L}} P_{x x}\left(e^{j\left(\frac{\Omega_{\mathrm{d}}}{L}-\frac{2 \pi n}{L}\right)}\right)}{P_{x_{\mathrm{d}} x_{\mathrm{d}}}\left(e^{j \Omega_{\mathrm{d}}}\right)}
$$

Proof: See Appendix A.

This is a general expression of the optimal reconstruction filter when $P_{x x}$ is known.

\section{APPLICATION TO A FIRST-ORDER AUTOREGRESSIVE PROCESS}

Assume that the process $x[n]$ is a stationary $\mathrm{AR}(1)$ process,

$$
x[n]=a x[n-1]+v[n],
$$


where $a$ is a constant, $|a|<1$. The signal is downsampled with a factor $L$ into $x_{\mathrm{d}}[n]=x[n L]$. It is then easy to show that $x_{\mathrm{d}}[n]$ is also an $\mathrm{AR}(1)$ process,

$$
x_{\mathrm{d}}[n]=a_{\mathrm{d}} x_{\mathrm{d}}[n-1]+v_{\mathrm{d}}[n],
$$

where $a_{\mathrm{d}}=a^{L}$ and $v_{\mathrm{d}}=\sum_{r=0}^{L-1} a^{r} v[L n-r]$. The spectra of the $\mathrm{AR}(1)$ processes are,

$$
\begin{gathered}
P_{x x}\left(e^{j \Omega}\right)=\frac{\sigma_{v}^{2}}{\left(1-a e^{-j \Omega}\right)\left(1-a e^{j \Omega}\right)}, \\
P_{x_{\mathrm{d}} x_{\mathrm{d}}}\left(e^{j \Omega_{\mathrm{d}}}\right)=\frac{\sigma_{v}^{2} \sum_{r=0}^{L-1} a^{2 r}}{\left(1-a^{L} e^{-j \Omega_{\mathrm{d}}}\right)\left(1-a^{L} e^{j \Omega_{\mathrm{d}}}\right)},
\end{gathered}
$$

where $\Omega_{\mathrm{d}}=\Omega L$. Inserting (7) and (8) into (4) leads to the following result,

Proposition 2. Let $x[n]$ be an AR(1) process that is sampled into $x_{\mathrm{d}}[n]=x[n L]$. Then the MMSE optimal interpolation filter to reconstruct the signal $x[n]$ from the samples $x_{\mathrm{d}}[n]$ is given by

$$
h[n]=c_{1} \delta[n+1]+c_{0} \delta[n],
$$

where

$$
\begin{aligned}
& c_{1}=\frac{1}{1-a^{2 L}} a^{L-k}\left(1-a^{2 k}\right), \\
& c_{0}=\frac{1}{1-a^{2 L}} a^{k}\left(1-a^{2 L-2 k}\right),
\end{aligned}
$$

i.e., the filter depends only on the neighbouring samples.

Proof: See Appendix B.

Since the AR(1) process is Markovian, it is reasonable that the optimal interpolation filter only needs to consider information from the two adjacent points. While downsampled AR(1) processes are still AR(1), this is unfortunately not the case for higher-order AR processes, thereby limiting the generalisation of Proposition 2.

\subsection{Comparison to linear interpolation}

According to (9), the filter produces interpolation points by a weighted mean of the previous and the following sample point. Note the close relationship between the AR(1) filter and ordinary linear interpolation, defined as

$$
x[L n+k]=\frac{(L-k)}{L} x_{\mathrm{d}}[n]+\frac{k}{L} x_{\mathrm{d}}[n+1],
$$

and for the $\mathrm{AR}(1)$ interpolation filter,

$$
\begin{aligned}
x[L n+k] & =\frac{1}{1-a^{2 L}} a^{k}\left(1-a^{2 L-2 k}\right) x_{\mathrm{d}}[n] \\
& +\frac{1}{1-a^{2 L}} a^{L-k}\left(1-a^{2 k}\right) x_{\mathrm{d}}[n+1] .
\end{aligned}
$$

Fig.1 shows that the coefficients of the AR(1) filter are damped versions of the linear interpolation coefficients. The AR(1) filter considers the stochastic nature of the signal and introduces a bias towards the mean, which in this case is zero. The larger $a$ is (i.e., less stochasticity), the more our filter resembles linear interpolation.

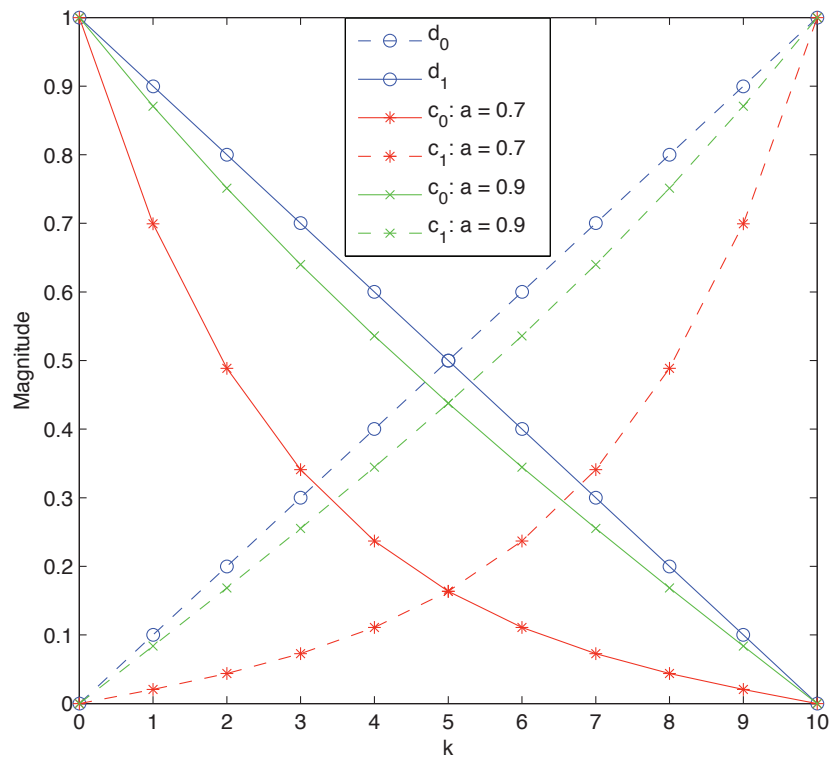

Fig. 1. The coefficients for linear interpolation $\left(d_{0}=k / L\right.$, $\left.d_{1}=(L-k) / L\right)$ and the coefficients for the AR(1) filter in (9) with $L=10$.

\section{LOCAL AR APPROXIMATION}

Unfortunately, real world signals are not always well approximated as stationary AR(1) processes. In order to extend the above results and to take advantage of the simplicity of the interpolation filter, we propose to model an arbitrary signal locally as an AR(1) process, in other words to use a non-stationary AR(1) model.

We propose an algorithm where the $a$-parameter in (5) is optimised locally. This is done by first estimating $a_{\mathrm{d}}$ with a weighted prediction error method [8], i.e.,

$$
\hat{a}_{\mathrm{d}}=\arg \min _{a_{\mathrm{d}}} f\left(a_{\mathrm{d}}\right),
$$

where

$$
f\left(a_{\mathrm{d}}\right)=\sum_{m=0}^{N-1} w(m)\left(x_{\mathrm{d}}[m]-a_{\mathrm{d}} x_{\mathrm{d}}[m-1]\right)^{2} .
$$

The weights, $w(m)$, are selected as a Gaussian window, centered at the time of the interpolation point $\left(n_{k}=n+k / L\right)$,

$$
w(m)=e^{\frac{-\left(m-n_{k}\right)^{2}}{2 \alpha^{2}}},
$$

where $\alpha$ is the standard deviation of the Gaussian window. We call the algorithm local AR approximation (LARA), since it can be seen as a stochastic version of the LPA method [6], the latter being better suited for deterministic signals.

Since $a_{\mathrm{d}}=a^{L}$, we attain $a=a_{\mathrm{d}}^{1 / L}$. To obtain a unique solution for $a$, negative values are excluded. This is equivalent to assuming that both processes are of a low-pass character. Note that this assumption is only necessary for signals with unknown spectra. 


\section{NUMERICAL EXPERIMENTS AND RESULTS}

In this section, we test the AR(1) filter and the LARA algorithm. We generate discrete signals, upsample and interpolate them, then compute the resulting MSEs. To facilitate the comparison between different processes, the MSEs are normalised by the variance of the signal.

\section{1. $\operatorname{AR}(1)$ filter experiments}

First, we compare the resulting MSEs when interpolating a true $\mathrm{AR}(1)$ signal with the AR(1) filter and the linear interpolation filter. Three different $\mathrm{AR}(1)$ processes are investigated. Each signal is 10.000 samples long and a normalised MSE is computed from 250 Monte Carlo simulations. We confirm that the derived AR(1) filter is indeed better than linear interpolation, see Table 1 . The difference is higher the smaller $a$ is.

\subsection{LARA filter experiments}

Secondly, we investigate the performance of LARA. The processes are generated, downsampled and interpolated with LARA and other interpolation techniques. The MSE is computed over 500 Monte Carlo simulations, where each signal consists of 3.000 samples. The first process is an $\mathrm{AR}(2)$ process,

$$
x[n]=0.3 x[n-1]+0.4 x[n-2]+v[n],
$$

where $v$ is white Gaussian noise, $\mathcal{N}(0,1)$. The resulting normalised MSE, for different $L$, is compared to other interpolation techniques: linear, spline and LPA. The parameters of LPA are set to $m=1$ and $h=0.02 L$. The standard deviation, $\alpha$, of the weight function for LARA (see (16)), is set to 20 samples $(\alpha=20 L)$. These parameter settings were empirically selected to minimise the interpolation MSE for the evaluated interpolation techniques. Fig. 2 shows the resulting MSE for the different interpolation techniques. In this case, the optimal LPA is indistinguishable from linear interpolation, and both are outperformed by LARA.

To see how the LARA algorithm performs when the process is not stationary, we investigate the result for an $\mathrm{AR}(1)$ process where the pole is changing with time according to $a=\sin (2 \pi n 200 / T)$, where $T$ is the time between two samples in $x$. The results are shown in Fig. 3. As seen, the performance of LARA is clearly better than that of the other interpolation methods for both simulated processes, especially for large $L$.

Table 1. MSE for linear and AR(1) filter interpolation of three $\operatorname{AR}(1)$ processes

\begin{tabular}{|l|c||c|c|c|c|c|}
\hline Method & $a$ & $L=2$ & $L=3$ & $L=4$ & $L=5$ & $L=8$ \\
\hline AR(1) & 0.5 & 0.30 & 0.48 & 0.59 & 0.67 & 0.79 \\
Linear & 0.5 & 0.31 & 0.52 & 0.68 & 0.80 & 1.05 \\
\hline AR(1) & 0.7 & 0.17 & 0.29 & 0.39 & 0.47 & 0.64 \\
Linear & 0.7 & 0.17 & 0.30 & 0.41 & 0.50 & 0.73 \\
\hline AR(1) & 0.9 & 0.05 & 0.09 & 0.13 & 0.17 & 0.26 \\
Linear & 0.9 & 0.05 & 0.09 & 0.13 & 0.17 & 0.27 \\
\hline
\end{tabular}

\section{CONCLUSIONS AND FUTURE WORK}

We have derived a MMSE optimal interpolation filter for a discrete signal with a known spectrum. We have explicitly derived this filter

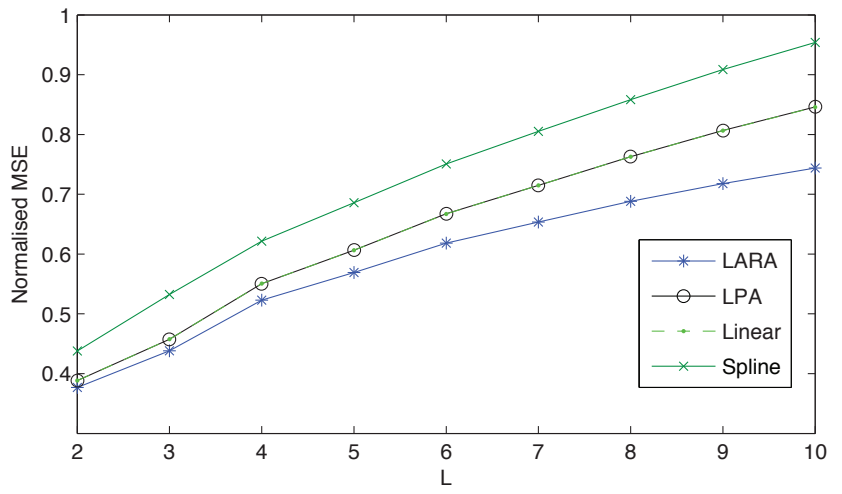

Fig. 2. Normalised MSE for interpolating the AR(2) process in (17) with LARA algorithm, linear and spline interpolation.

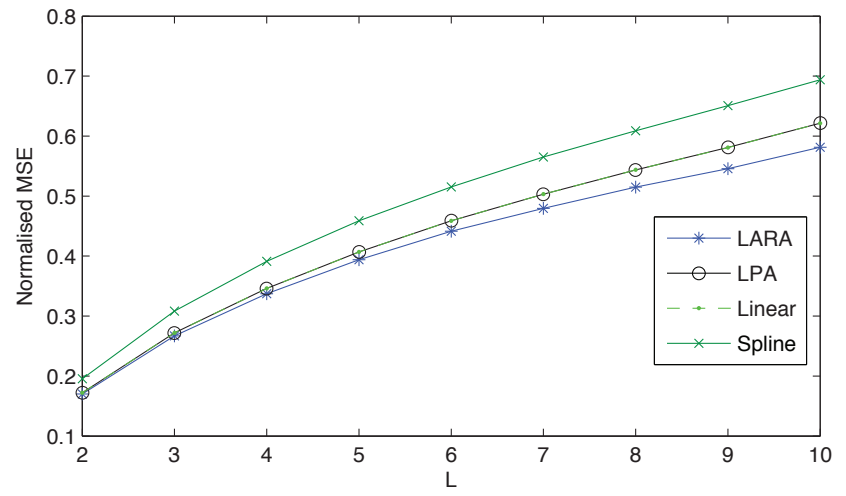

Fig. 3. Normalised MSE when applying LARA, LPA, linear and spline interpolation on an $\mathrm{AR}(1)$ process with varying parameter, $a=\sin (2 \pi n 200 / T), T=0.1$.

for an $\mathrm{AR}(1)$ process and shown that it is similar to a linear interpolation filter, but biased towards the mean.

We also introduce the LARA algorithm, which locally approximates an unknown process as an AR(1) process. Numerical experiments show examples of processes where the LARA algorithm outperforms commonly used interpolation techniques. The filter resulting from the LARA algorithm depends crucially on the width of the Gaussian window, $\alpha$. Currently, the selection of $\alpha$ is done empirically. Therefore, the obvious next step in developing the LARA algorithm is to propose a theoretical approach for the selection. Furthermore, it needs to be more thoroughly investigated for which processes the algorithm is effective.

\section{APPENDIX}

\section{A. PROOF OF PROPOSITION 1}

The spectrum $P_{x_{\mathrm{d}} x_{\mathrm{dk}}}$ is the Fourier transform of $R_{x_{\mathrm{d}} x_{\mathrm{dk}}}[\tau]=$ $E\left[x_{\mathrm{d}}[n] x_{\mathrm{dk}}[n]\right]$, which after reformulation yields,

$$
P_{x_{\mathrm{d}} x_{\mathrm{dk}}}\left(e^{j \Omega_{\mathrm{d}}}\right)=\sum_{\tau=-\infty}^{\infty} R_{x x}[\tau L+k] e^{-j \Omega_{\mathrm{d}} \tau},
$$


where

$$
R_{x x}[\tau L+k]=R_{x x}\left[\tau^{\prime}\right] \sum_{\tau^{\prime}=-\infty}^{\infty} \delta\left[\tau^{\prime}-n L-k\right] .
$$

This yields

$$
P_{x_{\mathrm{d}} x \mathrm{dk}}=e^{j \frac{\Omega_{\mathrm{d}} k}{L}} \sum_{\tau^{\prime}=-\infty}^{\infty} R_{x x}\left[\tau^{\prime}\right] \sum_{\tau^{\prime}=-\infty}^{\infty} \delta\left[\tau^{\prime}-n L-k\right] .
$$

Expressing the pulse train as its Fourier series results in

$$
\begin{aligned}
P_{x_{\mathrm{d}} x_{\mathrm{dk}}} & =e^{j \frac{\Omega_{\mathrm{d}} k}{L}} \sum_{\tau=-\infty}^{\infty} R_{x x}[\tau] \frac{1}{L} \sum_{n=<L>} e^{\frac{j n 2 \pi}{L}(\tau-k)} e^{-j \frac{\Omega_{\mathrm{d}} \tau}{L}} \\
& =\frac{e^{j \Omega_{\mathrm{d}} k / L}}{L} \sum_{n=<L>} e^{\frac{-j n 2 \pi k}{L}} P_{x x}\left(e^{j\left(\frac{\Omega_{\mathrm{d}}}{L}-\frac{n 2 \pi}{L}\right)}\right) .
\end{aligned}
$$

Inserting (18) into (3) now yields (4).

\section{B. PROOF OF PROPOSITION 2}

Inserting (7)-(8) into (4) results in

$$
H_{\mathrm{k}}(z)=\frac{\frac{1}{L} z^{\frac{k}{L}}}{\sum_{r=0}^{L-1} a^{2 r}} \sum_{m=0}^{L-1} \frac{e^{-j \frac{2 \pi m k}{L}} \prod_{i \in\{-1,+1\}}\left(1-a^{L} z^{i}\right)}{\prod_{i \in\{-1,+1\}}\left(1-a z^{\frac{i}{L}} e^{j \frac{i 2 \pi m k}{L}}\right)},
$$

where $z=e^{j \Omega_{\mathrm{d}}}$. Equation (20) can be simplified by noting that

$$
\left(e^{j 2 \pi k m / L}\right)^{L}=1,
$$

and, from inverting a geometric sum $(x \neq 1)$,

$$
1-x^{L}=(1-x) \sum_{m=0}^{L-1} x^{n} .
$$

Using (20) and (21), we can rewrite the terms in the numerator (let $x=a z^{\frac{i}{L}} e^{j \frac{i 2 \pi m k}{L}}$ ) and (19) becomes

$$
\begin{aligned}
H_{\mathrm{k}}(z) & =\frac{\frac{1}{L} z^{\frac{k}{L}}}{\sum_{r=0}^{L-1} a^{2 r}} \sum_{m=0}^{L-1} e^{-\frac{j 2 \pi m k}{L}} \sum_{n=0}^{L-1}\left(a z^{-\frac{1}{L}} e^{j \frac{2 \pi m}{L}}\right)^{n} \\
& \cdot \sum_{p=0}^{L-1}\left(a z^{\frac{1}{L}} e^{-j \frac{2 \pi m}{L}}\right)^{p} \\
& =\frac{\frac{1}{L} z^{\frac{k}{L}}}{\sum_{r=0}^{L-1} a^{2 r}} \sum_{n, p, m} a^{n+p} z^{\frac{-n+p}{L}} e^{\frac{j 2 \pi m}{L}(-k+n-p)} .
\end{aligned}
$$

The sum over $m$ is $L$ if $n-k-p=\kappa L, \kappa \in \mathbb{Z}$, and zero otherwise. This yields

$$
\begin{aligned}
H_{\mathrm{k}}(z) & =\frac{1}{\sum_{r=0}^{L-1} a^{2 r}}\left[a^{-k+L} z \sum_{\tilde{p}=0}^{k-1} a^{2 \tilde{p}}+a^{k} \sum_{p=0}^{L-1-k} a^{2 p}\right] \\
& =\frac{1}{1-a^{2 L}}\left(z a^{L-k}\left(1-a^{2 k}\right)+a^{k}\left(1-a^{2 L-2 k}\right)\right) .
\end{aligned}
$$

Taking the inverse Z-transform shows (9)-(11).

\section{REFERENCES}

[1] Y. C. Eldar and T. Michaeli, "Beyond bandlimited sampling," IEEE Signal Processing Mag., vol. 26, no. 3, pp. 48-68, May 2009.

[2] M. Unser, "Sampling - 50 years after Shannon," Proc. IEEE, vol. 88, no. 4, pp. 569-587, Apr. 2000.

[3] M. B. Matthews, "On the linear minimum-mean-squared-error estimation of an undersampled wide-sense stationary random process," IEEE Trans. Signal Processing, vol. 48, no. 1, pp. 272-275, Jan. 2000.

[4] T. M. Lehmann, C. Gonner, and K. Spitzer, "Survey: Interpolation methods in medical image processing," IEEE Trans. Pattern Anal. Mach. Intell., vol. 18, no. 11, pp. 1049-1075, Nov. 1999.

[5] S. V. Vaseghi and P. J. W. Rayner, "A new application of adaptive filters for restoration of archived gramophone recordings," in Proc. Int. Conf. on Acoust., Speech and Signal Process., 1988, vol. 5, pp. 2548-2551.

[6] V. Katkovnik, K. Egiazarian, and J. Astola, Local Approximation Techniques in Signal and Image Processing, SPIE Press, Bellingham, Washington, 2006.

[7] A. Papoulis, Signal Analysis, McGraw-Hill Companies, 1977.

[8] L. Ljung, System Identification: Theory for the user, PrenticeHall, Upper Saddle River, NJ, 2nd edition, 1999. 